\title{
Modeling Technology and Research of Digital Manufacturing System of Automobile Parts Based on Multi-Agent System
}

\author{
Shi Pengyao ${ }^{1}$, Zhao Ying ${ }^{1}$ and Xiao Mingya ${ }^{2}$ \\ (1.NanChang Institute of Science and Technology, Nanchang,330108; Jiangxi Engineering \\ Laboratory of Digital Manufacturing for Automobile Parts; 2. AnHui QuanChai Engine CO.,LTD)
}

\begin{abstract}
Keywords: Multi-Agent System; Automotive Parts; Digital Manufacturing System; Modeling Technology
\end{abstract}

\begin{abstract}
Combining the traditional automobile and motorcycle parts production workshops to develop networking and digitization, based on the production characteristics of automobile and motorcycle parts production workshops with multiple varieties, small batches and customization, this paper proposed a digitization of automobile and motorcycle parts production workshops, explained the basic connotation and overall implementation framework of digital manufacturing in automobile and motorcycle parts production workshops, proposed the key technology system of digital manufacturing model, studied the intelligent analysis and adaptation of multi-source information in workshops, and integrated networked devices based on embedded intelligent terminals and other key technologies. Finally, combined with the above research results, a digital manufacturing support system for automobile and motorcycle parts production workshops was designed and developed, and it was applied in a typical automobile and motorcycle parts production workshop and achieved good application results.
\end{abstract}

\section{Introduction}

In order to improve the performance of manufacturing system production control, a hybrid control model based on multi-agent system was established. The model divides the production control system into a management agent layer, a unit agent layer, and an execution agent layer. The management agent layer is responsible for scheduling and coordinating the various unit agents and managing all the agents. The unit agents in the unit agent layer cooperate with each other through the common database. The agent is responsible for the hardware in the manufacturing system. According to the local local resource information and the current state, the published task is received and solved. There is a distributed structure between the same level of agents. Adopting a multi-agent based hybrid control mode improves the real-time and flexibility of manufacturing system production control. The validity of the model was verified.

\section{Digital Manufacturing Technology}

In layman's terms, digitization is the transformation of many complex and varied information into measurable numbers and data, and the establishment of appropriate digital models using these numbers and data, transforming them into a series of binary codes, introducing them into the computer, and unifying them. Processing, this is the basic process of digitization. With the development of computer technology, humans can use the extremely simple " 0 " and " 1 " coding techniques for the first time to encode and decode all sounds, texts, images and data. The collection, processing, storage and transmission of various types of information have achieved standardization and high-speed processing. Digital manufacturing refers to the digitization of manufacturing. It is the result of the intersection, integration, development, and application of manufacturing technology, computer technology, network technology, and management science. It is also inevitable that manufacturing companies, manufacturing systems, production processes, and production systems are constantly digitizing. The trend includes three levels: design-centered digital manufacturing technology, control-centered digital manufacturing technology, and management-centered digital manufacturing technology. 


\section{Production Line Layout Design and Optimization}

In the automotive industry, the production line is made up of equipment and operational staff and is the basic management unit for the production of finished products, parts or products. The production efficiency of the production line directly affects the level of production costs. Increasing the efficiency of the production line is an effective means of reducing production costs. Automotive parts and components are mass-produced. The basic requirements of the production line are high-efficiency, high-quality, low-consumption materials and flexible green manufacturing. The layout design of the production line is divided into the following four steps:

Preliminary planning. According to the process specification and production plan, the system layout design method (SLP method) is used to comprehensively consider the limitations of the actual layout and the effects of their interactions. The type and quantity of equipment required for the production line are initially calculated and the initial design is obtained. Program. Among them, when designing the overall layout of a factory using the SLP method, it is necessary to clarify the relationship between the logistics and information flow among various units and draw a diagram of the relationship.

Detailed Planning. Based on the previous work, according to the construction space, process constraints and the size of the equipment, consider the auxiliary service facilities that may be involved in buffers and warehouses of the transport equipment. Under the actual constraints, with the help of the Factory CAD software tools, detailed information can be obtained. The two-dimensional layout of the production line.

Modeling and Simulation. According to the layout plan of the production line, the object model of each entity on the production line is first established, including machine tools, buffer stations, workers, material transportation equipment, pallets, etc., and then the camshaft storage equipment and transportation are defined according to the material transportation route and the conditions of the transportation equipment. Methods, etc., define all Activity Points, set the Material Handling Device of the camshaft production line, and then use the Move Arrow to use the production process flow, handling equipment, and production route. All the activity points are connected to form a from-to roadmap. Finally, the logistics system is simulated by the Factory FLOW software for Euclid, rectilinear, and actual algorithms.

Optimization Evaluation. The completion of the previous work has laid a solid data foundation for the optimization evaluation, including equipment utilization, production idle time, production efficiency, material handling costs and worker work efficiency, as well as the number of finished products, etc., selecting the appropriate evaluation model for each program Comprehensive comparison optimization, choose the best layout program.

\section{Production Control Model Based on Multi-agent Manufacturing System}

Around the application of MAS in the manufacturing field, extensive research has been conducted. Generally, the subsystems of the manufacturing system are regarded as an independent unit to be studied. The integration between the units of the manufacturing system is not considered. In view of this, this paper builds a manufacturing system production control model that integrates multiple production units based on the M AS technology. The model organically combines multiple intelligent modules and physical entities to form a certain intelligent control system. Through the analysis of the model, the production control can be optimized to accurately complete the complex production tasks. According to the structural characteristics of the manufacturing system, the established agent model is shown in Figure 


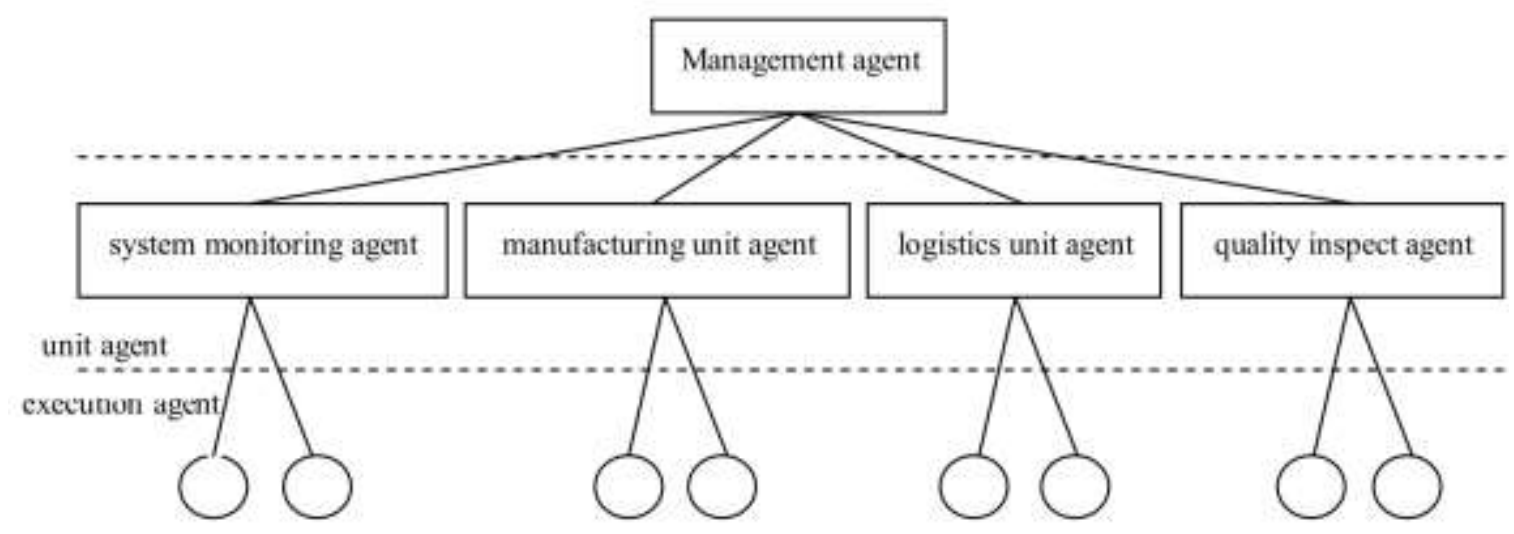

Fig 1 MAS-based manufacturing system production control model

The MAS model consists of a management agent layer, a unit agent layer, and an execution agent layer. The management agent is responsible for maintaining and updating the MAS. It receives task input through the man-machine interface and generates task planning and release. The unit agent layer defines the system monitoring agent, processing unit agent, and logistics unit intelligence according to the characteristics of the manufacturing system. The body and quality inspection agent is a class of four-unit agent. Each unit agent receives the task issued by the management agent and re-issued. The agent performs the task and solves according to the local information and the current status.

The MAS-based manufacturing system production control model shown in Fig. 1 can decentralize the decision-making capability of the entire system into each agent so as to reduce the system's centralized control capability, improve the system's ability to respond to unexpected failures, and system-to-information Real-time capabilities of processing. Through in-depth analysis of Figure 1, it is found that the model is actually a hybrid control architecture, which combines the characteristics of hierarchical hierarchies and distributed structures.

\section{Conclusion}

For the layout and planning of auto parts production lines, based on the design of the production line, according to the actual situation of the company, the digital modeling and simulation of the production line will be carried out so that the company can directly and visually see the site environment on the shop floor and obtain a digital analysis report. The optimization of the production line provides a theoretical basis, which in turn realizes the purpose of reducing costs, increasing production efficiency, and obtaining better economic benefits. This article applies MAS to the production control system of modern automobile manufacturing systems, and establishes a multi-agent production control model integrating logistics, processing, quality management, and information monitoring. Compared with the centralized control mode, the established M AS control model improves the real-time capability of the manufacturing system.

\section{Acknowledgement}

Project funding: Project at Nanchang institute of science and technology (No.SZZX-17-01)

\section{References}

[1] Elmaraghy H A, Algeddawy T. Co-evolution of products and manufacturing capabilities and application in auto-parts assembly[J]. Flexible Services \& Manufacturing Journal, 2012, 24(2):142-170.

[2] Matsumoto M, Ikeda A. Examination of demand forecasting by time series analysis for auto parts remanufacturing[J]. Journal of Remanufacturing, 2015, 5(1):1. 
[3] Wang Y, Chang X, Chen Z, et al. Impact of subsidy policies on recycling and remanufacturing using system dynamics methodology: a case of auto parts in China[J]. Journal of Cleaner Production, 2014, 74(7):161-171.

[4] Gan Y, Guangying L I, Ming tu M A, et al. Development of advanced compact steel process and deep working technology for high-strength-ductility auto-parts[J]. Steel Rolling, 2015.

[5] Jian-Cheng D A, Zhou X Y, Zhou T, et al. Research Status and Prospects of Surface Strengthening Technology of Auto Parts[J]. Surface Technology, 2015.

[6] Abdulrahman D A, Subramanian N, Liu C, et al. Viability of remanufacturing practice: a strategic decision making framework for Chinese auto-parts companies[J]. Journal of Cleaner Production, 2015, 105(1):311-323.

[7] Dussel P E. The Auto Parts-Automotive Chain in Mexico and China: Co-operation Potential?[J]. China Quarterly, 2012, 209(209):82-110.

[8] Türkcan K, Ates A. Vertical Intra-industry Trade and Fragmentation: An Empirical Examination of the US Auto-parts Industry[J]. World Economy, 2011, 34(1):154-172.

[9] Matsumoto M, Ikeda A. Examination of demand forecasting by time series analysis for auto parts remanufacturing[J]. Journal of Remanufacturing, 2015, 5(1):1.

[10] Matsumoto M, Ikeda A. Examination of demand forecasting by time series analysis for auto parts remanufacturing[J]. Journal of Remanufacturing, 2015, 5(1):1.

[11] Shen Y, Song Y, Hua L, et al. Influence of Plastic Deformation on Martensitic Transformation During Hot Stamping of Complex Structure Auto Parts[J]. Journal of Materials Engineering \& Performance, 2017, 26:1-9.

[12] Matsumoto M, Chinen K, Endo H. Remanufactured Auto Parts Market in Japan: Historical Review and Factors Affecting Green Purchasing Behavior[J]. Journal of Cleaner Production, 2017. 